\title{
Nematic Order in Ferrofluids
}

\author{
H. Pleiner ${ }^{1}$, E. Jarkova ${ }^{1}$, H.-W. Müller ${ }^{1}$, and H.R. Brand ${ }^{2}$ \\ ${ }^{1}$ Max-Planck-Institute for Polymer Research, POBox 3148, 55021 Mainz, Germany \\ ${ }^{2}$ Theoretische Physik III, Universität Bayreuth, 95440 Bayreuth, Germany
}

(J. Magn. Magn. Mat. 252 (2002) 147)

With the help of a Landau free energy we discuss the phase transition from an isotropic ferrofluid to a nematic one. The latter can be either superparamagnetic or ferromagnetic. The transitions are first order and the ferromagnetic state can be reached directly from the isotropic one or via the superparamagnetic nematic one. An external magnetic field induces magnetic as well as nematic order. The transition to the ferronematic state is then via an imperfect bifurcation switching from a state of low nematic and magnetic order to one with strong nematic order and either (still) low or high magnetic order. In the latter case the director is either parallel or perpendicular to the external field (and the magnetization parallel to it) or all three directions are different (but lie in a common plane). Some hydrodynamic features of these phases are listed.

\section{Phase transitions without field}

The suspension of ferromagnetic nanoparticles in water or oil is known as a ferrofluid [1]. It is isotropic and paramagnetic, i.e. there is no spontaneous magnetic ordering (no spontaneous magnetization) in equilibrium. However, the particles can be oriented very easily in an external magnetic field leading to a large magnetization even for small fields. This superparamagnetic behavior is the basis for many applications. If the ferroparticles are suspended in a nematogen, there is an interplay of nematic and magnetic ordering. In principle, 4 combinations of nematic order/disorder with magnetic order/disorder are possible.

In order to investigate these possibilities we used a simple Landau-type free energy function [2] to describe the phase transition behavior. In the absence of an external magnetic field we find a first order transition from an unmagnetized isotropic ferrofluid (no magnetic, no nematic order) to either a superparamagnetic ferronematic (nematic, but no magnetic order) or to a ferromagnetic phase, where both, nematic $\left(S_{F}\right)$ and magnetic order $(M)$, are present.

As a result we find that a phase with ferromagnetic order but no nematic order is not possible in the context of the present model, since the former always implies the latter (but not vice versa). Depending on the coupling between nematic and magnetic order, the transition from the isotropic ferrofluid to the ferromag-

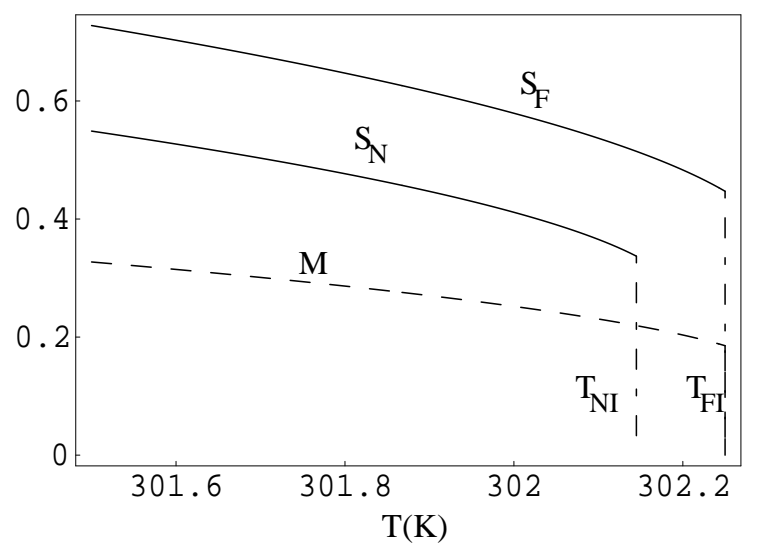

Figure 1: The discontinuous direct isotropic to ferromagnetic nematic $\left(S_{F} \neq 0 \neq M\right)$ transition at $T_{F I}$. The transition to the superparamagnetic state $\left(S_{N} \neq 0\right.$ and $\left.M=0\right)$ is suppressed, if $T_{N I}<$ $T_{F I}$.

netic nematic phase can be either direct (Fig. 1) or via the superparamagnetic ferronematic one (Fig. 2). In the ferromagnetic phase the orientation of the nematic director is either parallel or perpendicular to the magnetization. This phase has been discussed on the basis of a microscopic model long time ago [3], but seems not to have been found in nature until now.

\section{Phase transitions in a field}

An external field $\boldsymbol{H}$ induces a finite magnetization in the ferrofluid by orienting the 


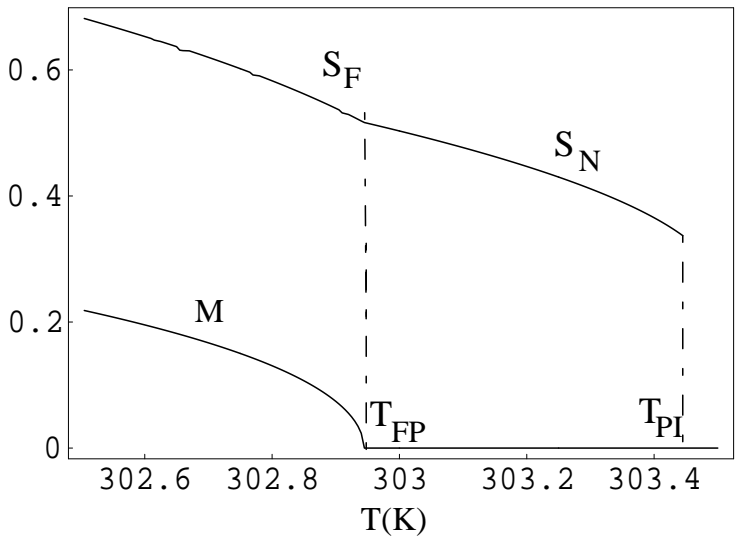

Figure 2: The intermediate superparamagnetic nematic state $\left(S_{N} \neq 0\right.$ and $\left.M=0\right)$ between $T_{P I}$ and $T_{F P}$.

magnetic particles. In addition it also orients the mesogens due to the diamagnetic anisotropy effect. Thus, any phase has a finite nematic and a finite magnetic order due to the external field, even if it is in the high temperature state. The transitions are then from low to high values of the nematic order and/or magnetization. This is described by the Landau free energy containing the nematic order parameter $Q_{i j}=\frac{1}{2} S\left(3 \hat{n}_{i} \hat{n}_{j}-\delta_{i j}\right)$ and the magnetization $\boldsymbol{M}=M \hat{\boldsymbol{m}}$

$$
\begin{aligned}
f & =f_{0}+\frac{A}{2} Q_{i j} Q_{i j}-\frac{B}{3} Q_{i j} Q_{j k} Q_{k i} \\
& +\frac{C_{1}}{4}\left(Q_{i j} Q_{i j}\right)^{2}+\frac{C_{2}}{4} Q_{i j} Q_{j k} Q_{k l} Q_{l i} \\
& +\frac{\alpha}{2} \boldsymbol{M}^{2}+\frac{\beta}{4} \boldsymbol{M}^{4}+\frac{\gamma}{2} M_{i} M_{j} Q_{i j} \\
& +\frac{\delta_{1}}{2} \boldsymbol{M}^{2} Q_{i j} Q_{i j}+\frac{\delta_{2}}{2} M_{i} M_{k} Q_{i j} Q_{k j} \\
& -\boldsymbol{M} \cdot \boldsymbol{H}-\frac{\chi_{S}}{2} H_{i} H_{j} Q_{i j}+\frac{\delta_{3}}{2} \boldsymbol{H}^{2} Q_{i j} Q_{i j} \\
& +\frac{\delta_{4}}{2} H_{i} H_{k} Q_{i j} Q_{k j}
\end{aligned}
$$

where $f_{0}$ is the free energy density of the isotropic (superparamagnetic) ferrofluid phase. The changes of sign of $A \equiv A_{0}\left(T-T_{N I}^{*}\right)$ and $\alpha \equiv \alpha_{0}\left(T-T_{F P}^{*}\right)$, at the critical temperatures $T_{N I}^{*}$ and $T_{F P}^{*}$, correspond to the hypothetical second order isotropic to nematic, and to the para- to ferromagnetic transition, respectively, if the cross coupling terms $\left(\sim \gamma\right.$ and $\left.\sim \delta_{1,2}\right)$, the cubic term $(\sim B)$, and the field are absent. All other coefficients, as well as $A_{0}$ and $\alpha_{0}$, are assumed to be constant near the transition point.

The equilibrium phases are minima of (1), where the partial derivatives of $f$ are zero. The independent variables, on which $f$ depends, are $S, M$, and the angles of the director $\hat{\boldsymbol{n}}$ relative to the field $\boldsymbol{H}$ and the magnetization direction $\hat{\boldsymbol{m}}$.

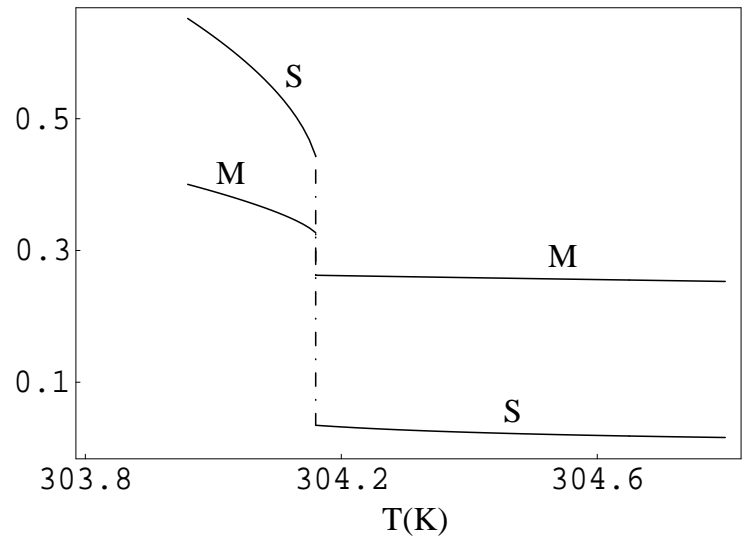

Figure 3: The transition under moderate external fields.

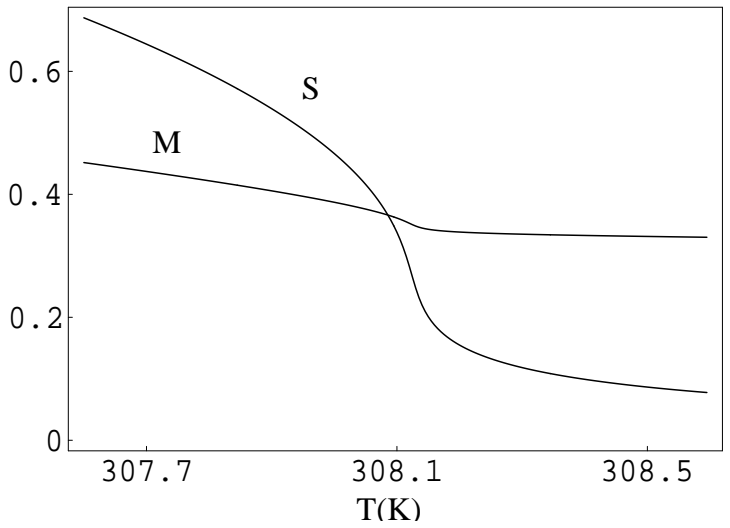

Figure 4: The transition under strong external fields is continuous.

For the orientations there are 3 cases: The director can be either parallel or perpendicular to the external field (in which cases the magnetization is parallel to the field). In addition, it is also possible that $\hat{\boldsymbol{n}}, \boldsymbol{H}$, and $\hat{\boldsymbol{m}}$ lie in a common plane, but are separated by finite angles, a case that has not yet been detected experimentally. For the first of these cases the transition from the low order phase (small $S$ and small $M$ ) to the high order phase (large $S$ and large $M$ ) is shown for a moderate (Fig.3) and a high (Fig.4) external field using reasonable values for the Landau parameters. Compared to the field-free case (Fig.1) the jumps in the magnetization and in the nematic order are reduced and shifted to higher temperatures. Eventually, for very strong external fields, they are replaced by smooth passages from low to high values (Fig.4). This field dependence of the nematic order resembles the isotropic-nematic transition in the presence of an external electrical field. In conventional nematics this behavior under an external magnetic field has never been observed, since the strong fields necessary are out- 
side the experimental reach, while in ferronematics the expectation is that the necessary magnetic fields are much smaller and thus this effect is detectable.

In an external magnetic field the superparamagnetic and the ferromagnetic nematic phases are identical in their symmetry properties and differ only quantitatively in the value of the magnetization. These phases are subject to increasing experimental and theoretical studies usually invoking external fields (e.g. [4, 5]). A clear distinction, however, is possible for vanishing external field, only.

\section{Hydrodynamics}

In an accompanying contribution [6] we discuss the hydrodynamics of the superparamagnetic nematic phase. Due to the behavior of magnetic fields under time reversal, linear field effects are possible in the dynamics only, but not in the statics. For heat and particle currents due to temperature and concentration gradients, for the relaxation of the nematic director into its equilibrium orientation, in the flow alignment due to shear flow, and in the stress tensor due to flow, such dynamic effects exist. Possible experiments to detect these new effects are discussed [6].

\section{Induced chaining}

In a strong external field ordinary ferrofluids (and magnetorheological fluids) can show formation of particle chains. These structures resemble nematic order, but do not have a nematic-like dynamics, since they do not exist without an external field. They can have internal degrees of freedom, either undulational or compressional. The latter renders the sound velocity anisotropic and gives rise to an additional slow mode [7].

\section{Acknowledgements}

Partial support of this work through the Schwerpunktsprogramm 1104 'Colloidal Magnetic Fluids' of the Deutsche Forschungsgemeinschaft is gratefully acknowledged.

\section{References}

[1] R.E. Rosensweig, Ferrohydrodynamics, (Cambridge University Press, New York, 1985).

[2] H. Pleiner, E. Jarkova, H.-W. Müller, H.R. Brand, Magnetohydrodynamics, 37 (2001) 254.
[3] F. Brochard and P.G. de Gennes, J. Phys. (France) 31 (1970) 69.

[4] Yu. Raikher and V.I. Stepanov, J. Magn. Magn. Mater. 201 (1999) 182.

[5] C.Y. Matuoa and A.M. Figueiredo Neto, Phys. Rev. E60 (1999) 1815.

[6] E. Jarkova, H. Pleiner, H.-W. Müller, A. Fink, H.R. Brand, Eur. Phys. J. E 5 (2002) 583.

[7] H.R. Brand and H. Pleiner, J. Magn. Magn. Mat. 85 (1990) 125 and Phys. Rev. Lett. 86 (2001) 1385 . 